\title{
A Dimensioning and Tolerancing Methodology for Concurrent Engineering Applications I: Problem Representation
}

M. N. ISLAM

Department of Mechanical Engineering, Curtin University of Technology, Perth, WA 6845, Australia 


\title{
A Dimensioning and Tolerancing Methodology for Concurrent Engineering Applications I: Problem Representation
}

\begin{abstract}
This paper is the first of two which present a methodology for determining the dimensional specifications of all the component parts and sub-assemblies of a product, according to their dimensional requirements. To achieve this goal, two major steps are followed, each of which is described in a paper. In the first paper, all relationships necessary for finding the values of dimensions and tolerances are represented in a matrix form, known as a Dimensional Requirements/Dimensions $(\mathrm{DR} / \mathrm{D})$ matrix. In the second paper, the values of individual dimensions and tolerances are determined by applying a comprehensive solution strategy to satisfy all the relationships represented in the DR/D matrix. The methodology is interactive and suitable for use in a Concurrent Engineering (CE) environment. The graphical tool presented in this paper will assist a CE team in visualizing the overall D\&T problem and foreseeing the ramifications of decisions regarding the selection of dimensions and tolerances. This will assist the CE team to systematically determine all the controllable variables, such as dimensions, tolerances, and manufacturing processes.
\end{abstract}

Keywords: Dimensioning and Tolerancing, Concurrent Engineering, Functional Dimension, Dimensional Specification 


\section{Introduction}

Dimensioning and Tolerancing (D\&T) has long been a topic of extensive research due to its relationship with all facets of manufacturing, including functionality, cost, quality and reliability. A competitive advantage can be obtained through the proper selection of dimensions and tolerances. However, despite all research efforts, the theories have not been applied in practice, and tolerances are still assigned based on past experiences, best guesses, or handbook data.

In recent years, a number of tolerance analysis software packages such as Vis VSA [1], CETOL [2], and DCS [3] have been released commercially, but these have not resolved the problem either. These packages often apply statistical tolerancing theories (Monte Carlo simulation has been found to be the most popular of these) to find the combined effect of the input tolerances (part tolerances) on the assembly tolerance. In principle, they could be used for "what-if" analysis; however, they generally lack an intervention strategy. Consequently, when the prime objective is to determine part tolerances based on the product's dimensional requirements, they are not appropriate for establishing dimensional specifications.

This paper is the first of two which present a methodology for determining the dimensional specifications of all the component parts and sub-assemblies of a product according to their dimensional requirements. A schematic representation of the methodology is illustrated in Figure 1. The methodology provides a systematic way of converting a product's dimensional requirements into product specifications. The methodology involves two major steps, each of which is described in a paper. In the first paper, all relationships necessary for solving D\&T problems are represented in a matrix form, known as a Dimensional Requirements/Dimensions (DR/D) matrix. In the second paper, the values of individual dimensions and tolerances are determined by satisfying all the relationships represented in a DR/D matrix. This is achieved by applying a comprehensive solution strategy that acts in conjunction with a generic tolerance allocation strategy. The methodology is interactive and suitable for use in a Concurrent Engineering (CE) environment. 
Figure 1: Schematic Representation of the Methodology.

\section{Dimensioning and Tolerancing Issues}

Over the years, an enormous amount of research has been published on D\&T, which makes it practically impossible to present an exhaustive review of this topic; hence, only the issues relevant to this paper are discussed here. Comprehensive treatments of the topic can be found in Chase and Parkinson [4] and Hong and Chang [5] with 104 and 270 reference papers cited, respectively.

Soon after World War II, the "Inter-Service Committee for Dimensioning and Tolerancing of Drawings" in the United Kingdom set down the Basic Principles for Dimensional Analysis of Engineering, the details of which are available in [6]. These principles are intended to provide the designer with tools to perform a logical analysis of a design. A D\&T methodology should be based on these principles.

One major area of D\&T research focused on the development of models of tolerance accumulation. The adopted model of tolerance accumulation is the basis of tolerance calculation because it represents a mathematical model that estimates the combined effect of component part tolerances on assembly tolerance. Various tolerance accumulation models are available, e.g. Worst Case (WC) model [7], Root Sum Square (RSS) model [7], Mean Shift model [8], Six Sigma model [9] and Monte Carlo model [10]. A comparison of these tolerance accumulation models and others are presented by Wu et al [11]. The methodology presented in this paper applies the WC accumulation model because it guaranties the interchangeability requirement, and it is also the most widely used model in the industry; however, if required, the proposed methodology can be modified to include other tolerance accumulation models.

There are two major approaches to D\&T [12, 13]: (i) tolerance analysis and (ii) tolerance allocation. In tolerance analysis, the component part tolerances are known, and the resulting assembly tolerance is calculated. Conversely, in 
tolerance allocation, the acceptable tolerances for an assembly are known, and the component part tolerances are calculated to meet the requirements. Therefore, at the design stage, the designer mainly encounters tolerance allocation problems. It is worth clarifying that, although the theme of this series of papers is tolerance allocation, the first paper primarily deals with representation and management of a number of interrelated tolerance allocation problems; the second paper will present a comprehensive strategy for finding the values of dimensions and tolerances through tolerance allocation.

Another area of D\&T research is tolerance specification, which is concerned with the specification of tolerance types and values. The specified tolerances must conform to relevant international [14], national [15, 16] and company standards. A number of concepts have been reported such as Features and Relations used in Object Orientated Modelling (FROOM) [17], Technologically and Topologically Related Surfaces (TTRS) [18-20], Proportioned Assembly Clearance Volume (PACV) [21], tolerancing for function [22], and functional tolerancing based on positioning features [23]. This paper follows the concept of functional D\&T, the details of which are available in [22, 24, 25].

From the review of current D\&T practices, two major deficiencies have been identified: (i) there is an interdisciplinary problem which has been tackled only by the designers and (ii) a systematic method for solving the problem is lacking. To overcome the interdisciplinary nature of the problem, the Concurrent Engineering (CE) approach has been adopted by a number of researchers [26-28]. Farmer [27] addresses the second deficiency by developing a matrix format in which he represents the D\&T problem of a panel guide mechanism. The methodology presented in this series of papers expands and enhances this idea.

The example used to illustrate the proposed methodology is a gear pump design in which it is assumed that the conceptual design has been finalized. The selected conceptual design drawing of the pump adopted from [29] is given in Figure 2. It is also assumed that the dimensional requirements of the gear pump have been identified and analysed, and their target values finalized (Table 1). The details of extracting dimensional requirements from the customer needs for the 
gear pump, and setting their target values are available in [30]. For the sake of simplicity, not all the gear pump dimensional requirements are included in Table1.

Figure 2: Gear Pump Assembly [29].

Table 1: List of Dimensional Requirements and Their Target Values of a Gear Pump [30].

\section{The Traditional Approach}

Traditionally, from the product design drawing, a designer identifies the critical parameters that require control for proper functioning of the design. These are known as functional requirements, acknowledging their relationship with the function of the product. The functional requirements, which are dimensional in nature, are the dimensional requirements of the product. The designer sets the target values for each dimensional requirement based on experimental results, analytic solutions, handbook data, or past experience.

The dimensional requirements usually depend on a number of individual dimensions and, therefore, the dimensional requirements are also known as dependent functional dimensions [31] or sum dimensions [32]. To guarantee the function of the product, the designer first identifies the dimensions that directly affect each dimensional requirement from the design drawing, and then establishes the geometric relationships between each dimensional requirement and the functional dimensions affecting that requirement. This relationship is known as a functional equation [33] or a fundamental equation [32]. A general functional equation can be expressed as follows:

$$
Z=f\left(X_{1}, X_{2}, \ldots \ldots, X_{n}\right)
$$

where, $Z$ is the dimensional requirement, and $X_{i}$ are the functional dimensions. 
The system of analysis for deriving functional equations is known as geometric analysis, details of which can be found in [31]. The currently available tolerance analysis software packages are capable of generating the relationships by taking data from 3D CAD models. Once the relationships are known, the numerical values of the functional dimensions are determined through tolerance allocation.

One such requirement for the gear pump example is "Control the gap between the width of Gear 1 and Body 8”, and the target value for the gap set by the designer is $(0.32 \pm 0.265) \mathrm{mm}$ (Table 1$)$. The three dimensions that directly affect the gap are identified from the design drawing (Figure 2). These dimensions are the depth of the recess, the thickness of the gasket, and the width of the gear (Figure 3). The designer indicates these dimensions in a symbolic form on a part drawing. Then, by applying a geometric analysis methodology, a functional equation, known as loop equation [31], can be established between the dimensional requirement and the related dimensions and tolerances. The equation is given below:

$$
(Z \pm z)=(L 81 \pm l 81)+(L 61 \pm l 61)-(L 11 \pm l 11)
$$

where, $(Z \pm z)$ is the gap between the width of the gear and body, (L11 \pm 11$)$ is the width of the gear, ( $L 61 \pm 161)$ is the thickness of the gasket, and $(L 81 \pm 181)$ is the depth of the recess.

Figure 3: Identification of Functional Dimensions.

This relationship can be used to find the values of the associated functional dimensions. However, an acceptable solution for the gear pump design must satisfy all dimensional requirements listed in Table 1, and the solution of each dimensional requirement requires a number of iterations. Therefore, it would be useful to represent the relationships necessary to solve D\&T problems in matrix form. A matrix will illustrate interactions between each set of items, viz. 
dimensional requirements, dimensions and tolerances, and process capability tolerances.

\section{Proposed Methodology}

The proposed methodology applies a feature-based parametric model for product definition. As such, dimensions and tolerances are initially denoted in a symbolic form, and the relationships between each dimensional requirement and the dimensions and tolerances are established. These relationships are then represented in a matrix format, and finally, the values of dimensions and tolerances are determined by satisfying all the relationships represented in the matrix.

In today’s engineering design environment, CE can only be implemented by means of computer-based systems [34]. Hence, the proposed methodology is developed with its future computer-based application in mind. Consequently, the required data can be imported directly from CAD files, thus diminishing the need for manual entry.

A flow-chart to express the different steps involved in the first part of the proposed methodology is illustrated in Figure 4. The methodology begins with a dimensional requirements list (Table 1) and a product design drawing (Figure 2).

Figure 4: Overview of the Methodology.

\subsection{Formation of a Preliminary DR/D Matrix}

The formation of a Dimensional Requirements/Parts matrix, which will eventually expand to the dimension level, is proposed; it is called a Preliminary Dimensional Requirements/ Dimensions (DR/D) matrix. This matrix is formed by listing the dimensional requirements down the left-hand side (1) and the part names across the top (2). After that, the parts that contribute to the fulfilment of a particular dimensional requirement are identified. If a relationship exists between a dimensional requirement and a part, then the symbol $X$ is entered into the 
Preliminary DR/D matrix (3). $X$ represents a simple relationship without indicating its strength. The construction of the Preliminary DR/D matrix ends when all the dimensional requirements have been considered (Figure 5).

Figure 5: Preliminary DR/D Matrix.

\subsection{Screening of the Preliminary DR/D Matrix}

The purpose of the screening process, (4) in Figure 4, is to identify the function of each part. This may lead to a reduction in the number of product parts. This is achieved by either eliminating a part, or by combining two or more parts into one. The reduction of the number of parts is one of the main pillars of the Design for Manufacturability (DFM) strategy. DFM is a core element of CE; for this reason, the part reduction strategy is included in the proposed methodology. Moreover, the reduction in the number of parts may be beneficial from a D\&T point of view since it often reduces the number of dimensions in a loop equation, enabling the tolerance values of the remaining dimensions in the loop to be increased. However, thought should be given to the costs involved, as a reduction in the number of parts usually increases the complexity of the remaining parts.

Another purpose of this screening process is to check whether all listed dimensional requirements are indeed dimensional in nature. If no relationship exists between a dimensional requirement and any part listed, then it should be eliminated.

\subsection{Establishment of Relationships between Dimensional Requirements and Dimensions and Tolerances}

After finalizing the Preliminary DR/D matrix, the next step is to extend each relationship to a dimension and tolerance level. To achieve this, the following procedure is proposed: 
- Identify the features that directly affect the dimensional requirements within each part (5). This procedure extends the relationship from each part to the feature level. However, it should be noted that the features have already been finalized during the configuration design stage.

- Within each feature, identify the dimensional elements (6) required by the function. These consist of the following dimensional elements: basic size, size tolerance, geometric tolerances, and surface texture parameter. The designer has to decide which dimensional elements need to be specified (7). This procedure extends the relationship from a feature level to a dimension and tolerance level. To perform this task, an in-depth knowledge of relevant standards, such as ISO 129-2004 [14], is required. Unfortunately, the standards do not prescribe a tolerance specification methodology [17]; hence, users must ensure that the appropriate standards have been followed.

- Dimensional element symbols are then entered (8) in the self-expanding DR/D matrix. Further details on the expanded DR/D matrix are given in Figure 6. It is worth noting that all the entries at the feature level are similar to the entries for the feature control frame, to be specified on the detailed drawing.

- Subsequently, in the expanded DR/D matrix, for each relationship between a dimensional requirement and a dimension and tolerance the symbol $X$ is entered, indicating a simple relationship without representing its strength.

- To facilitate further analysis, the D\&T problems are classified into different types, such as fitting feature problems, length dimension problems, geometric tolerancing problems, and centre distance problems. A detailed description of the types of problems involved, and the analysis techniques are given in [31]. The problem type is entered on the right-hand side of the matrix (9).

- The target values for the dimensional requirements are also entered in the right-hand side of the DR/D matrix (10).

- An equation representing the relationship between dimensional requirements and dimensions and tolerances is derived. This relationship, to be 
used for further analysis (11), is entered into the right-hand side of the DR/D matrix.

The final outcome of the above-described procedure, as applied to a dimensional requirement (DR1) from the gear pump example, is displayed in Figure 6. The Preliminary DR/D matrix, given in Figure 5, shows that the dimensional requirement DR1, i.e. "Control gap between Gear 1 Face and Body 8” is related to three parts, viz., Part 1: Gear, Part 6: Gasket and Part 8: Body. These relationships are then extended first to the feature level, and then to the dimension and tolerance level in the following manner.

First, the question is asked, "The dimensional requirement DR1 is related to Part 1, but which feature of Part 1 is related to DR1?" The answer is, "The width of the Gear, which is named Feature 11". Then the question is asked, "To represent the relationship between DR1 and Feature 11, which dimensional elements of Feature 11 should be specified?" The answer is, "Basic size L11 and linear tolerance \pm 111 ". In a similar manner, relationships between DR1 and Part 6 and Part 8 are analysed, prompting the entry of Feature 61 and Feature 81, and dimensions and tolerances $L 61 \pm l 61$ and $L 81 \pm 181$ into the DR/D matrix. Subsequently, the problem type, the relationship between the dimensional requirement and the dimensions and tolerances, and the target values are also entered into the DR/D matrix.

Figure 6: Expanded DR/D Matrix.

\subsection{Entering Details of Relationships into the DR/D Matrix}

As new dimensions and tolerances are entered into the DR/D matrix, they are grouped according to their parts. The build-up of the Part-Feature-Dimension hierarchy in the DR/D matrix (Figure 6) is worth noting because it is very useful from a manufacturing point of view. This phase of the methodology will end when information about all of the dimensional requirements has been entered. 


\subsection{Entering Details of Process Parameters into the DR/D Matrix}

The designer's objective is to satisfy the dimensional requirements by specifying dimensions and tolerances, while the manufacturing engineer simultaneously applies manufacturing processes to achieve the specified dimensions and tolerances. The designer, who is unable to see the consequences of his choices for tolerance values, cannot readily make changes. The designer, therefore, tends to act conservatively and safely. This often leads to tighter than necessary tolerances, known as fear tolerances*, which increase costs. On the other hand, a manufacturing engineer always tries to keep the tolerance values as wide as possible in an attempt to keep costs at a minimum. A CE environment avoids conflict between the designer and the manufacturing engineer since it includes representatives from both the design and the manufacturing departments. Since both points of views are present in the CE team, the requirements of both the designer and the manufacturing engineer can be considered simultaneously.

The proposed methodology introduces manufacturing requirements into the $\mathrm{DR} / \mathrm{D}$ matrix. The rationale behind this is to eliminate any miss-matches between the dimensions and tolerances (selected by the designer), and the manufacturing process capabilities (selected by the manufacturing engineer). The process parameters entered into the DR/D matrix (12) include the name of the proposed manufacturing process, its process capability, and the capability index for the manufacture of each feature. These process parameters will be used to calculate the process capability tolerance for each process used to manufacture each feature.

\footnotetext{
* This term was coined by Edward Roth, former president of the American Society of Mechanical Engineers [35].
} 


\subsection{Entering Proposed Tolerance Values into the DR/D Matrix}

Experienced designers tend to allocate tolerance values based on past experience, and while a formal methodology should always be encouraged, situations may arise where previous experiences will prove to be beneficial. Keeping this in mind, the proposed methodology initially allows some, or all, of the tolerance values proposed by the CE team (13) to be entered. In search of an optimum solution, these previously used tolerance values will have a higher priority than other tolerances in the system, although all the proposed tolerance values must be compatible with the proposed process capability tolerances.

\subsection{Completing the DR/D Matrix}

The DR/D matrix (Figure 7) is a multiple relationship matrix, which makes it possible to visually represent the interaction of a number of interrelated sets of items viz. dimensional requirements, dimensions and tolerances, and process capability tolerances. Besides these relationships, there are others which are not so apparent. Different types of relationships illustrated in the DR/D matrix (Figure 8) are described below. Numbers shown in $\#$ boxes refer to relationship types.

Along each row, dimensions and tolerances are tied to a dimensional requirement. This relationship is expressed by a loop equation and is controlled by the target value, which imposes performance constraints on the selection of dimensions and tolerances. For example, $R 2$ expresses a simple relationship between dimension requirement $D R 2$, and the dimensions related to it viz. (D12, $d 12 / 0)$ and $(D 13,0 /+d 13)$. The values of the dimensions and tolerances are controlled by the target value TV2 (see 1 in Figure 8).

Each chosen tolerance must satisfy the process capability requirements. This type of relationship imposes manufacturing constraints on the choice of tolerances. For example, tolerance $d 13$ must satisfy the process capability 
tolerance of Process 13 (see 2 in Figure 8). The following formula has been utilized by several authors [25, 31, 32, 36] to estimate the process capability tolerance achievable through various manufacturing processes.

$$
x \quad=\quad(0.45 \sqrt[3]{X}+0.001 X) 10^{\frac{I T-16}{5}}
$$

where $x$ is the process capability tolerance $(\mathrm{mm}), X$ is the manufactured dimension (mm), and IT is the International Tolerance grade number, a number that reflects the precision of the process.

Here it should be pointed out that, if reliable data is available from the shop floor, actual data should be entered instead of calculating the process capability tolerances.

All tolerances of a feature, viz. size and geometric tolerances, and surface texture parameters are controlled by the manufacturing process for that feature. For example, size tolerance $+d 13$ and surface texture parameter s13 are all produced by Process 13, and they should be compatible with the process capability tolerance and the surface texture capability (see 3 in Figure 8). Equation 3 shows an additional relationship between basic size and size tolerance. In most cases, geometric tolerances are also dependent on the maximum size of the feature.

Within a part, both the functional and non-functional dimensions are related through the parts process plan, and these relationships have profound effects on the manufacturing cost of the product (see 4 in Figure 8).

If one or more dimensions are shared by two or more functional equations, the functional equations are called coupled functional equations (see 5 in Figure 8). If none of the dimensions are shared by another functional equation, the functional equation is called an independent functional equation. In Figure 8, R3 is an example of an independent functional equation, and $R 1, R 4$ and $R 6$ are examples of coupled functional equations. It is interesting to note that, on the process level, functional equations $R 2$ and $R 5$ are also coupled functional 
equations. Here, the coupling follows the common feature (Feature 13), which is produced by a common manufacturing process (Process 13). Coupled dimensional requirements should be solved simultaneously, and the selected coupled tolerance value must match the process capability tolerance. For example, tolerance value $\pm l 11$ (Figure 8) should be the same for $D R 1, D R 4$ and $D R 6$, and it should be equal to, or less than, the process capability tolerance achievable through Process 11. When the coupling is on the process level, the process capability tolerance should match the geometric tolerance and/or surface texture parameter through which the coupling follows. For example, s13 and $+d 13$ (Figure 8) will be produced by the same process (Process 13), and the selected tolerance value $+d 13$ and surface texture parameter s13 should match the capability of Process 13.

Besides relationships among dimensional requirements, dimensions and tolerances, and process parameters, the DR/D matrix illustrates some other information viz. target values, relationships (loop equations), types of dimensional requirements, and target differences. The target difference is the difference between the value calculated by the dimensional constraints and the specified target value. The target difference column (not shown in Figure 7) will be helpful when determining the optimal values derived from the DR/D matrix. The relationship column will not be necessary once the methodology is integrated into a computer-based tool and interfaced with CAD software capable of calculating the assembly tolerances from individual dimensions and tolerances.

Figure 7: Complete DR/D Matrix for Gear Pump Design Example.

Figure 8: Types of Relationships Illustrated in DR/D Matrix.

\section{Discussion}

It is difficult to come up with a case that includes all the aspects of D\&T, and finding their solutions is time consuming. Thus, the gear pump example is chosen as a compromise between the complexity of a problem and the time required to 
find the solution. Here, only a 1D problem is presented because it can be formulated and solved manually. Complex calculations are required for formulating and solving multidimensional problems and several tolerance propagation concepts have been reported such as kinematic chain [37], small displacement torsor [38], Jacobian transforms [39], and tolerance maps [40]. In future a suitable tolerance propagation model has to be integrated with the proposed methodology to handle multidimensional problems.

The use of a matrix or spreadsheet format for organising D\&T problems is not new; designers have been using different custom-made formats for years. For example, CATS-1D [41] also adopted a spreadsheet format to organize data. CATS-1D is a freely available D\&T software package based on research carried out at Brigham Young University's ADCATS program. However, their representation is not meaningful from a manufacturing point of view because they employ an L-shaped matrix to represent two-factor relationships between dimensions and their nominal values, tolerances, distributions, capability indices, and others.

The proposed methodology is based on the matrix format developed by Farmer [27]; however, there are significant differences between the two approaches. These are outlined below:

The proposed methodology groups the dimensions on a part basis, where the relationships between a part, a feature, and the dimensions become visible in a hierarchical structure. Farmer [27] grouped the dimensions into size, geometry, surface texture, etc. This type of grouping has no practical significance as the dimensions will be produced as elements of a part. Moreover, grouping into size, geometry, surface texture etc. fails to represent the interrelationships between all the features and dimensions within each part (relationship type 4 in Figure 8) and the relationships between each feature and the processes used to manufacture (relationship type 3 in Figure 8). These relationships are very important for selecting proper manufacturing processes, because the selected process has to simultaneously satisfy all the requirements for size tolerance, geometric tolerances, and surface texture parameters. The consideration of part levels as a 
building block is also significant from an inspection point of view, because if any functional dimension is out of specification then that part will be rejected.

In the Function/Dimension matrix, Farmer [27] uses the symbol $X$ to register a simple relationship and numbers, e.g., 1 or -1 are used where a definite relationship exists. These numbers represent coefficients for individual dimensions in a loop equation, and they are only possible when the relationships are linear; however, in practice not all relationships are linear. Moreover, if the matrix uses numbers to represent the variable coefficients in the loop equation, then the same matrix cannot be used to solve the dimensioning problem, which requires a place to store the actual variable values after each iteration. For this reason, in the present methodology, $X$ is used to represent all types of relationships, and extra columns are added to store the actual relationships. This allows the proposed methodology to handle both linear and non-linear relationships.

After completing the representation of the D\&T problem, the next step is to find the values of individual dimensions and tolerances, indicated by $X$, in such a way that they satisfy all of the relationships built into the DR/D matrix. This is done through a number of iterations, which need a platform at each step to store the values. The DR/D matrix will be used for this purpose. In contrast, in the traditional approach, the designer selects one dimensional requirement at a time and finds its solution through several iterations, without having any clear idea of what is expected next. Because D\&T requires numerous trade-offs, it is always beneficial to have a complete picture of the problem, which the proposed methodology gives.

\section{Concluding Remarks}

- This paper presents the first part of a methodology to determine the dimension and tolerance values for a product from the dimensional requirements. The methodology provides a systematic and meaningful way of representing all the necessary relationships in a matrix format, known as DR/D matrix. 
- The newly developed DR/D matrix is a powerful tool that gives a complete picture of the complex relationships that must be satisfied to determine the dimensions and tolerances of the component parts and sub-assemblies of a product.

- All the relationships that must be satisfied are already built into the DR/D matrix; consequently, any violation of them will become immediately noticeable to the user.

- In the second paper, the DR/D matrix will be used as a platform for D\&T problems where results can be stored, crosschecked, and eventually optimized through an iterative process.

\section{References}

1. Vis VSA (2008) SIMENS US Site, Accessed through Internet (11/4/2008), http://www.plm.automation.siemens.com/en_us/products/tecnomatix/quality/vis_vsa.shtml

2. CETOL (2008) Sigmetrix Homepage, Accessed through Internet (11/4/2008), http://www.sigmetrix.com/default.asp

3. DCS (2008) Dimensional Control Systems Homepage Accessed through Internet (11/4/2008), http://www.3dcs.com

4. Chase KW, Parkinson AR (1991) A Survey of Research in the Application of Tolerance Analysis to the Design of Mechanical Assemblies, Research in Engineering Design, 3:23 -37

5. Hong YS, Chang TC (2002) A Comprehensive Review of Tolerancing Research, Int J Production Research, 40(11): 2425-2459

6. Dimensional Analysis of Engineering Designs (1948), Vol 1, Components, Part 1, His Majesty's Stationery Office, London

7. Fortini ET (1967) Dimensioning for Interchangeable Manufacture, Industrial Press, New York

8. Greenwood WH, Chase KW (1987) A New Tolerance Analysis Method for Designers and Manufacturers, J of Engineering for Industry, 109:112-116

9. Taylor WA (1991) Optimization and Variation Reduction in Quality, McGraw-Hill, Inc., New York

10. Sharpiro SS, Gross AJ (1981) Statistical Modelling Techniques, Marcel Dekker, Inc., New York

11. Wu Z, ElMaraghy WH, ElMaraghy HA (1988) Evaluation of Cost-Tolerance Algorithms for Design Tolerance Analysis and Synthesis, Manufacturing Review, 1(3):168 -179 
12. Chase KW, Greenwood WH (1988) Design Issues in Mechanical Tolerance Analysis, Manufacturing Review, 1(1):50 -59

13. Gerth RJ (1997) Tolerance Analysis: A Tutorial of Current Practice, in Zhang HC (Ed), Advanced Tolerancing Techniques, Chapter 4, John Wiley \& Sons, Inc., 65-99.

14. ISO 129-2004 (2004) Technical Drawings - Indication of Dimensions and Tolerances- Part 1, International Standards Organisation, Geneva

15. AS1100 (1984) Technical Drawing, Part 201-1984, Mechanical Drawing, The Standards Association of Australia, Sydney

16. ASME Y14.5-1994 (1999) Dimensioning and Tolerancing, ASME, 1999, Reaffirmed

17. Salomons OW, Poerink HJJ, Haalboom FJ, van Slooten F, van Houten FJAM, Kals HJJ (1996) A Computer Aided Tolerancing Tool I: Tolerancing Specification, Computers in Industry, 31:61-174

18. Clement A, Riviere A, Serre P, Valade C (1998) The TTRS: 13 Oriented Constraints for Dimensioning and Tolerancing, in ElMaraghy HA (Ed.), Geometric Design Tolerancing: Theories, Standards and Applications, 122-131

19. Dersrochers A, Clement A (1994) Dimensioning and Tolerancing Model for CAD/CAM Systems, Int. J. of Advanced Manufacturing Technology, 9:352-361

20. Kumaravel P, Anamd S, Ullas U, Mohanram V (2006) Cost Optimization of Process Allocation- A Tree Based Approach, Int. J. of Advanced Manufacturing Technology, 34:703-713

21. Teissandier D, Couetard Y, Gerard A (1999) A Computer Aided Tolerancing Model Proportioned Assembly Clearance Volume, Computer-Aided Design, 31:805-817

22. Weill R (1997) Dimensioning and Tolerancing for Function, in Zhang HC (Ed.), Advanced Tolerancing Techniques, John Wiley \& Sons, 329-354

23. Anselmetti B (2006) Generation of Functional Tolerancing Based on Positioning Features, Computer -Aided Design, 38:902-919

24. Weill R, Clement R, Hocken R, Farmer LE, Gladman CA, Wirtz A, Bourdet P, Freckleton JE, Kunzmann H, Ham I, Trumpold H, Matthias E (1988) Tolerancing for Function, Keynote Paper, Annals of the CIRP, 37(2):603-610

25. Farmer LE (1999) Dimensioning and Tolerancing for Function and Economic Manufacture, Blueprint Publ., Sydney, Australia

26. Wilhem RG, Lu SCY (1992) Tolerance Synthesis to Support Concurrent Engineering, Annals of the CIRP, 41(1):197-200

27. Farmer LE (1993) Function Oriented Dimensioning Enhances Concurrent Engineering Performance, Proceedings ACME’93, IE Aust., 171-175.

28. Islam M.N (2003) Concurrent Engineering Offers the Best Solution to Tolerance Allocation Problems, New Engineer J, 6 (1):14-15.16. 
29. Farmer LE, Gladman CC, Edensor KH. (1990) The Scope of Tolerancing Problems in Engineering, Report 1990/ITM/1, School of Mechanical and Industrial Engineering, The University of New South Wales, Sydney

30. Islam MN (2004) A Methodology for Extracting Dimensional Requirements for a Product from Customer Needs, Int. J. of Advanced Manufacturing Technology, 23: 489-494

31. Gladman CA (1972) Geometric Analysis of Engineering Designs, $2^{\text {nd }}$ Edition, Australian Trade Publications Pty. Ltd., Sydney, Australia

32. Bjørke Ø (1995) Computer-Aided Tolerancing, $2^{\text {nd }}$ Edition, ASME Press, New York

33. Benett G, Gupta LC (1969) Least-Cost Tolerances - I, Int J Production Research, 8 (1): 65-73

34. O’Flynn MJ, Ahmed MM (1993) Computer-Based Concurrent Engineering System, in Parsai HR, William G (Eds), Concurrent Engineering: Contemporary Issues and Modern Design Tools, Chapman and Hall, London, 184-206.

35. Tool and Manufacturing Engineers Handbook (1992) vol. VI, Design for Manufacturability, $4^{\text {th }}$ Edition, Society of Manufacturing Engineers, Dearborn

36. Conway HG (1966) Engineering Tolerances, $3^{\text {rd }}$ Edition, Sir Isaac Pitman \& Sons Ltd., London

37. Rivest L, Fortin C and Morel C (1994) Tolerancing a Solid Model with a Kinematic Formulation, Computer-Aided Design, 26: 465-476

38. Bourdet P, Mathieu L, Lartigue C and Ballu A (1996) The Concept of Small Displacement Torsor in Metrology, in Ciarlini P, Cox MG, Pavese F and Richter D (eds), Advances Mathematical Tools in Metrology II, World Scientific Publ. Co., 110-122

39. Laperriere L and El Maraghy HA (2000) Tolerance Analysis and Synthesis Using Jacobian Transforms, Annals of the CIRP, 49/1: 359-362

40. Bhide S, Ameta G, Davidson JK and Shah JJ (2007) Tolerance-Maps Applied to the Straightness and Orientation of an Axis, in Davidson, JK (ed), Models for Computer Aided Tolerancing in Design and Manufacturing, Springer, 45-54

41. CATS-1D (2008) Association for the Development of Computer-Aided Tolerancing Systems (ADCATS) Home Page, Accessed through Internet (11/4/2008) ,http://adcats.et.byu.edu/home.html, 
Table 1. List of Dimensional Requirements and their Target Values of a Gear Pump

\begin{tabular}{||l|l||}
\hline \multicolumn{1}{|c|}{ Dimensional Requirements } & \multicolumn{1}{c|}{ Target Values } \\
\hline DR01 Control gap between Gear 1 face and Body 8 & Gap $=0.32 \pm 0.265 \mathrm{~mm}$ \\
\hline DR02 Control gap between Gear 3 face and Body 8 & Gap $=0.32 \pm 0.265 \mathrm{~mm}$ \\
\hline DR03 Control gap between Gear 1 periphery and Body 8 & $0<$ Gap $\leq 0.4 \mathrm{~mm}$ \\
\hline DR04 Control gap between Gear 3 periphery and Body 8 & $0<$ Gap $\leq 0.4 \mathrm{~mm}$ \\
\hline DR05 Select fit for Bearing and Driving Shaft & Fit $=0.034 \pm 0.018 \mathrm{~mm}$ \\
\hline DR06 Select fit for Bearing and Driven Shaft & Fit $=0.034 \pm 0.018 \mathrm{~mm}$ \\
\hline DR07 Select fit for Cover hole and Driving Shaft & Loose running fit \\
\hline DR08 Select fit for Cover hole and Driven Shaft & Fit $=0.085 \pm 0.035 \mathrm{~mm}$ \\
\hline DR09 Select fit for Body hole and Bolt & Fit $=0.008 \pm 0.008 \mathrm{~mm}$ \\
\hline DR10 Selected fit for Gear 1 hole and Driving shaft & Fit $=-0.024 \pm 0.017 \mathrm{~mm}$ \\
\hline DR11 Selected fit for Gear 1 hole and Driven shaft & Fit $=-0.024 \pm 0.017 \mathrm{~mm}$ \\
\hline DR12 Select fit for Body and Locating Pins & Interference fit \\
\hline DR13 Control angular play of Locating Pins (Body) & $0<$ Angular play $\leq 3^{\prime}$ \\
\hline DR14 Select fit for Cover and Locating Pins & Close positional fit \\
\hline DR15 Control angular play of Locating Pins (Cover) & $0<$ Angular play $\leq 3^{\prime}$ \\
\hline DR16 Select fit for Stop and Body & Fit = 0.052 $\pm 0.052 \mathrm{~mm}$ \\
\hline DR17 Select fit for Stop and Driving Shaft & Slack running fit \\
\hline DR18 Select fit for Stop and Driving Pulley & Normal running fit \\
\hline DR19 Select fit for Body and Sealing Nut & H6g6 \\
\hline DR20 Select fit for Cover Hole and Bolt & Slack running fit \\
\hline DR21 Select fit for Cover Hole and Bolt Head & Slack running fit \\
\hline \hline
\end{tabular}




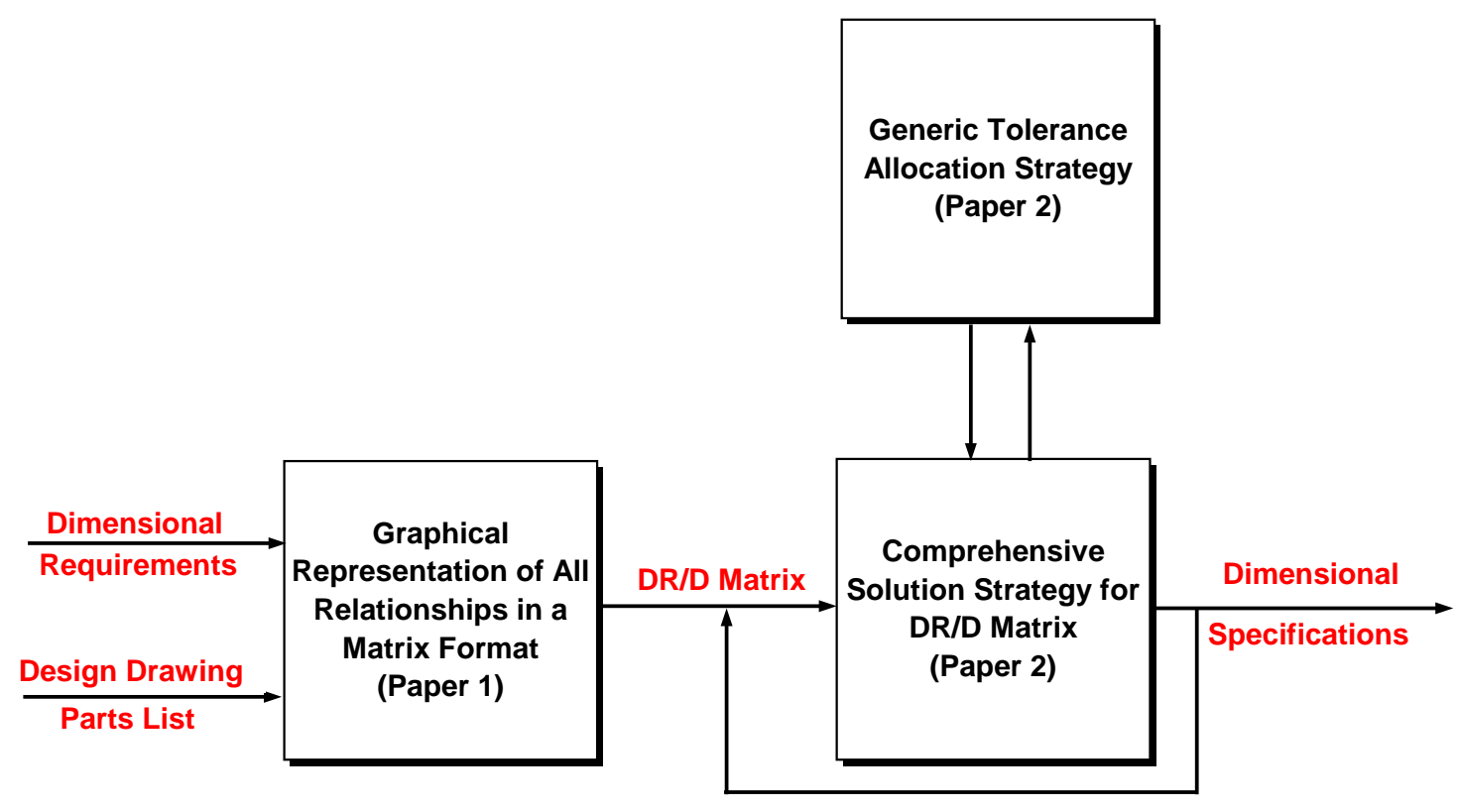

Figure 1 Schematic Representation of the Methodology 


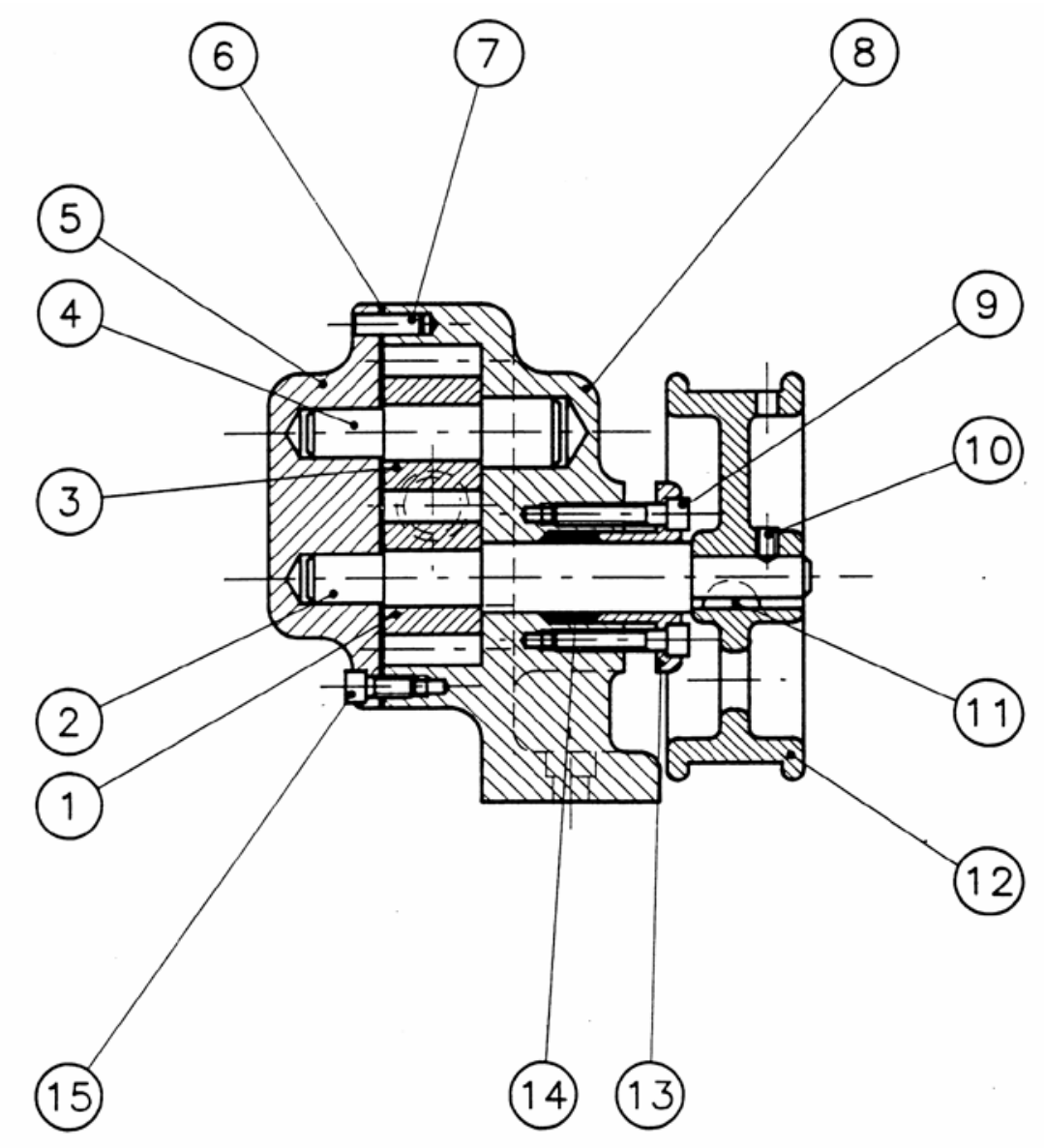

Figure 2. Gear Pump Assembly [29] 


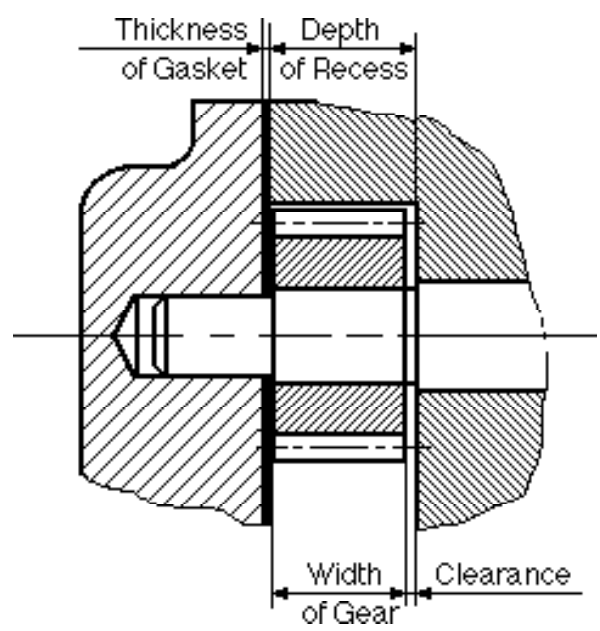

Clearance $=$ Depth of Recess + Thickness of Gasket Width of the Gear

Figure 3. Identification of Functional Dimensions 
(1)

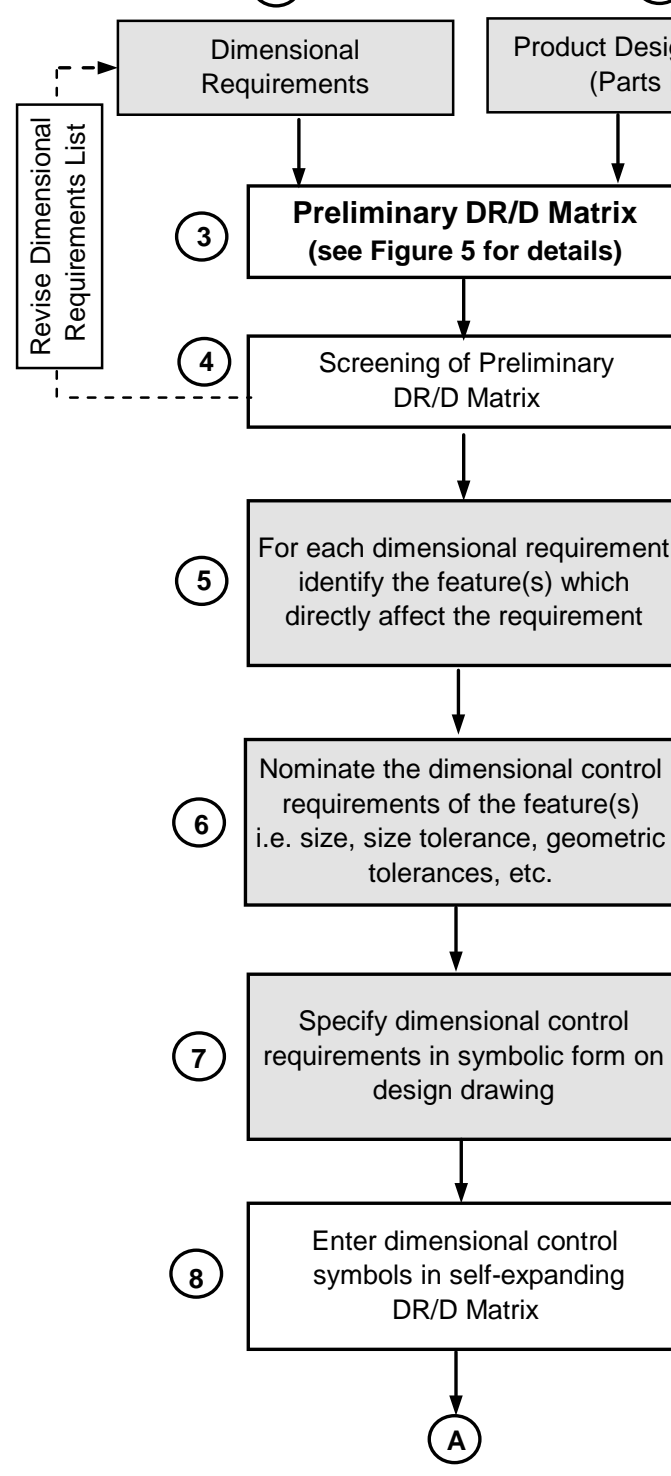

(2)

sign Drawing
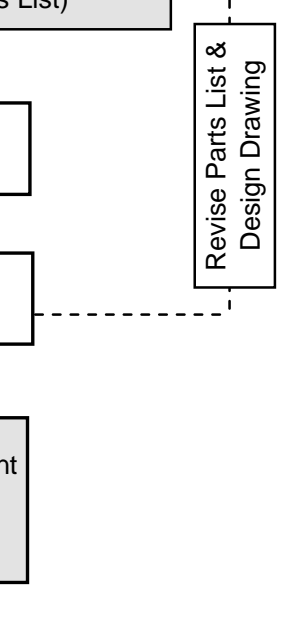

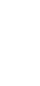




\begin{tabular}{|c|c|c|c|c|c|c|c|c|c|c|c|c|}
\hline \multirow{2}{*}{ Dimensional Requirements } & \multicolumn{11}{|c|}{ Parts List } & \multirow[b]{2}{*}{ 14. Stop } \\
\hline & 1. Gear & 2. Dr. Shaft & 3. Gear & 4. Dn. Shaft & 5. Cover & 6. Gasket & 7. Locating Pin & 8. Body & 9. Bolt| & 12. Pulley & 13. Sealing Nut & \\
\hline DR01 Control gap between Gear 1 face and Body 8 & $\mathrm{x}$ & & & & & $\mathrm{x}$ & & $\mathrm{x}$ & & & & \\
\hline DR02 Control gap between Gear 3 face and Body 8 & & & $\mathrm{x}$ & & & $\mathrm{x}$ & & $\mathrm{x}$ & & & & \\
\hline DR03 Control gap between Gear 1 periphery and Body 8 & $\mathrm{x}$ & $\mathbf{x}$ & & & & & & $\mathrm{x}$ & & & & \\
\hline DR04 Control gap between Gear 3 periphery and Body 8 & & & $\mathrm{x}$ & $\mathbf{x}$ & & & & $\mathrm{x}$ & & & & \\
\hline DR05 Select fit for Bearing and Driving Shaft & & $\mathbf{x}$ & & & & & & $x$ & & & & \\
\hline DR06 Select fit for Bearing and Driven Shaft & & & & $\mathrm{x}$ & & & & $\mathrm{x}$ & & & & \\
\hline DR07 Select fit for Cover hole and Driving Shaft & & $\mathbf{x}$ & & & $\mathbf{x}$ & & & $\bar{x}$ & & & & \\
\hline DR08 Select fit for Cover hole and Driven Shaft & & & & $\mathbf{x}$ & $x$ & & & & & & & \\
\hline DR09 Select fit for Body hole and Bolt & & & & & & & & $\mathrm{x}$ & $\mathrm{x}$ & & & \\
\hline DR10 Selected fit for Gear 1 hole and Driving shaft & $\mathrm{x}$ & $\mathrm{x}$ & & & & & & & & & & \\
\hline DR11 Selected fit for Gear 3 hole and Driven shaft & & & $\mathbf{x}$ & $\mathrm{x}$ & & & & & & & & \\
\hline DR12 Select fit for Body and Locating Pins & & & & & & & $\mathrm{x}$ & $\mathbf{x}$ & & & & \\
\hline DR13 Control angular play of Locating Pins (Body) & & & & & & & $x$ & $x$ & & & & \\
\hline DR14 Select fit for Cover and Locating Pins & & & & & $\mathrm{x}$ & & $\mathrm{x}$ & & & & & \\
\hline DR15 Control angular play of Locating Pins (Cover) & & & & & $\mathrm{x}$ & & $\bar{x}$ & & & & & \\
\hline DR16 Select fit for Stop and Body & & & & & & & & $\mathrm{x}$ & & & & $\mathrm{x}$ \\
\hline DR17 Select fit for Stop and Driving Shaft & & $\mathrm{x}$ & & & & & & & & & & $\mathrm{x}$ \\
\hline DR18 Select fit for Stop and Driving Pulley & & $x$ & & & & & & & & $\mathrm{x}$ & & \\
\hline DR19 Select fit for Body and Sealing Nut & & & & & & & & $x$ & & & $\mathrm{x}$ & \\
\hline DR20 Select fit for Cover Hole and Bolt & & & & & $\mathrm{x}$ & & & & $\mathrm{x}$ & & & \\
\hline DR21 Select fit for Cover Hole and Bolt Head & & & & & $\mathrm{x}$ & & & & $\mathrm{x}$ & & & \\
\hline
\end{tabular}

Figure 5. Preliminary DR/D Matrix 


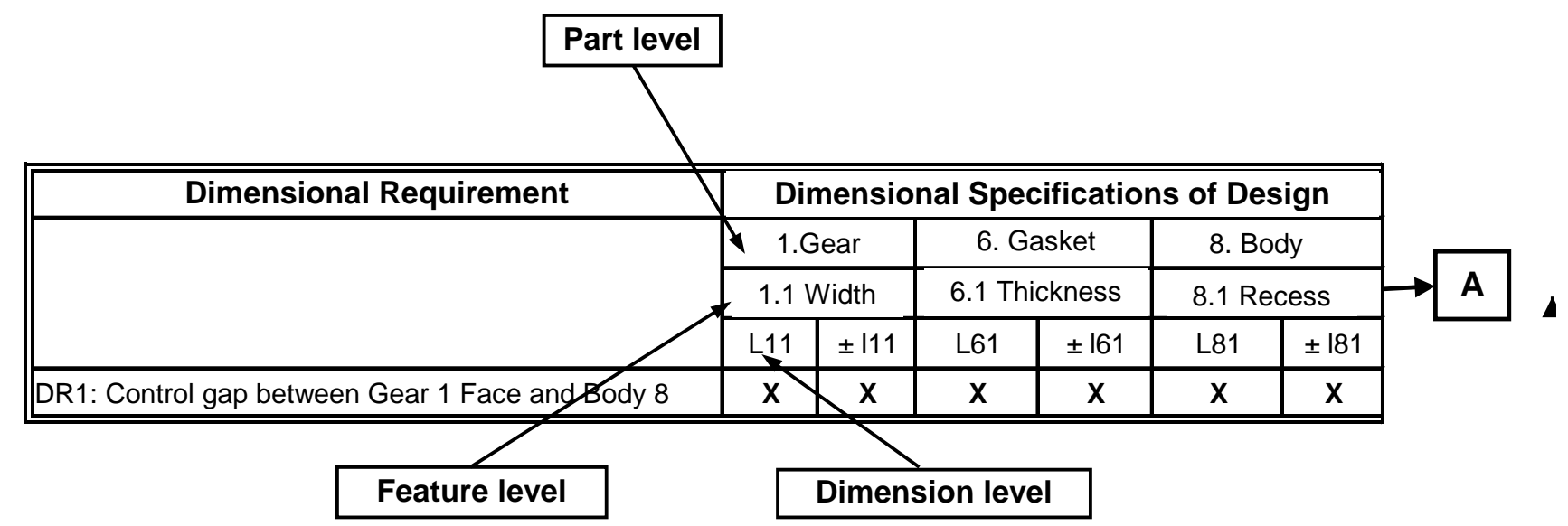

\begin{tabular}{|c|c|c|}
\hline \hline DR Type & Target Value & Relationship \\
\hline & & \\
\hline & & \\
\hline & & \\
\hline & & $(\mathrm{Z} \pm \mathrm{z})=(\mathrm{L} 81 \pm 181)+(\mathrm{L} 61 \pm \mathrm{I61})-(\mathrm{L} 11 \pm \mathrm{I11})$ \\
\hline
\end{tabular}

Figure 6. Expanding Relationships to Dimension and Tolerance Level 


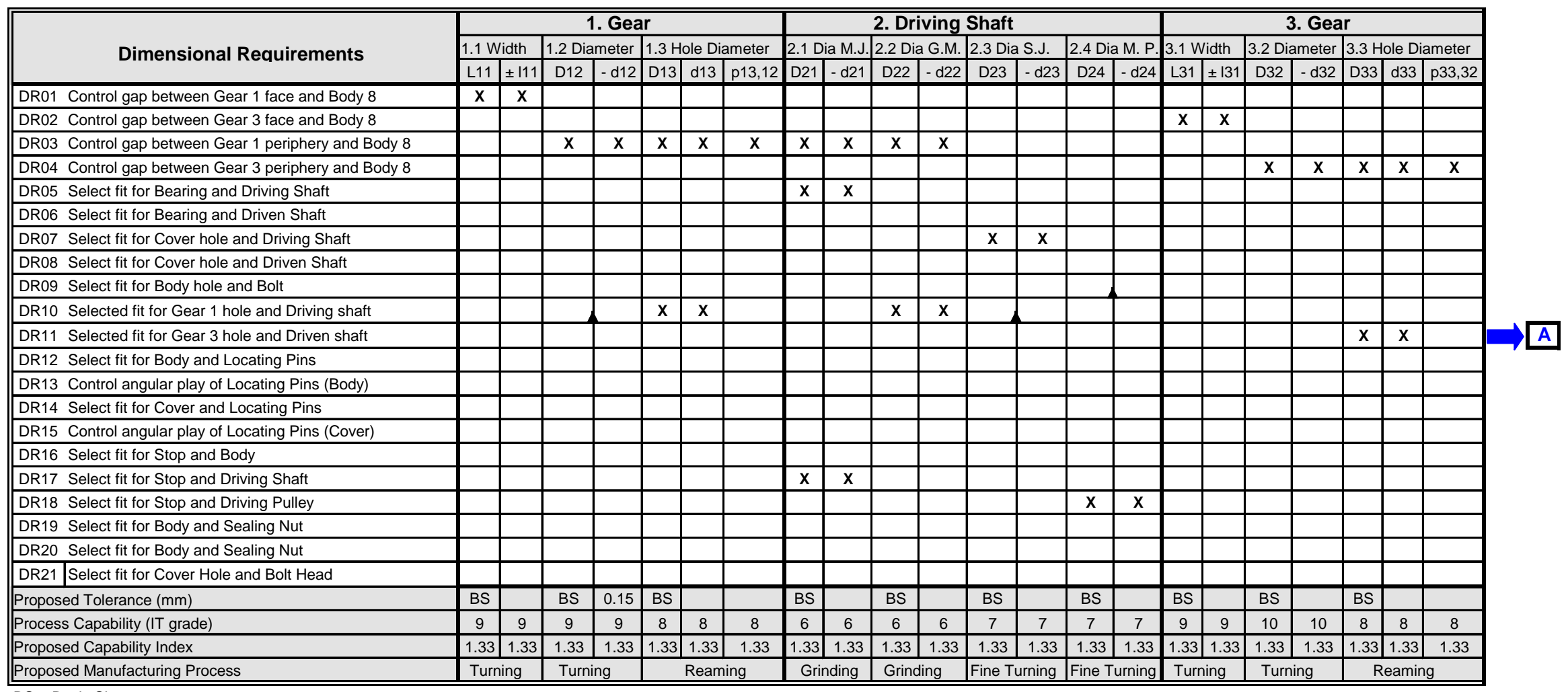

BS = Basic Size

Figure 7 . Complete DR/D Matrix 


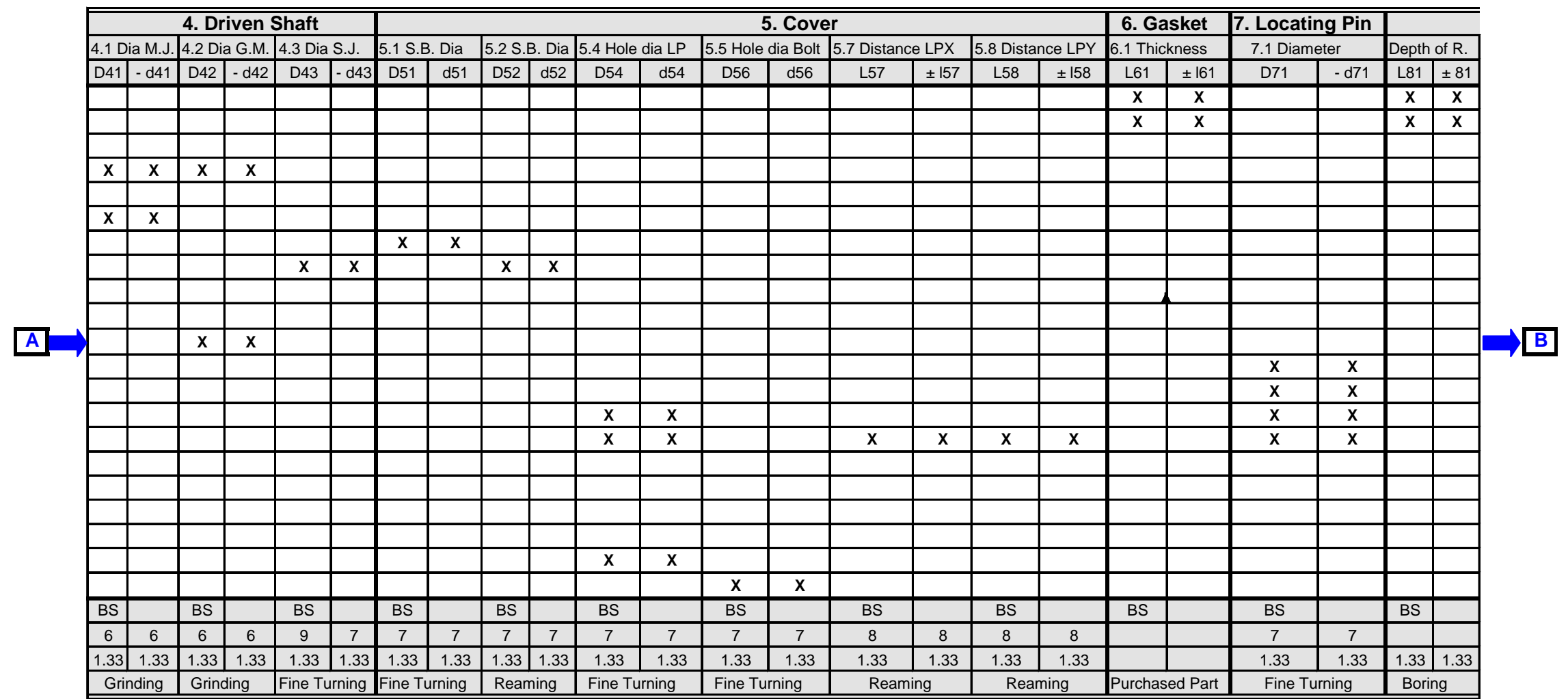

Figure 7 (Cotd). Complete DR/D Matrix 


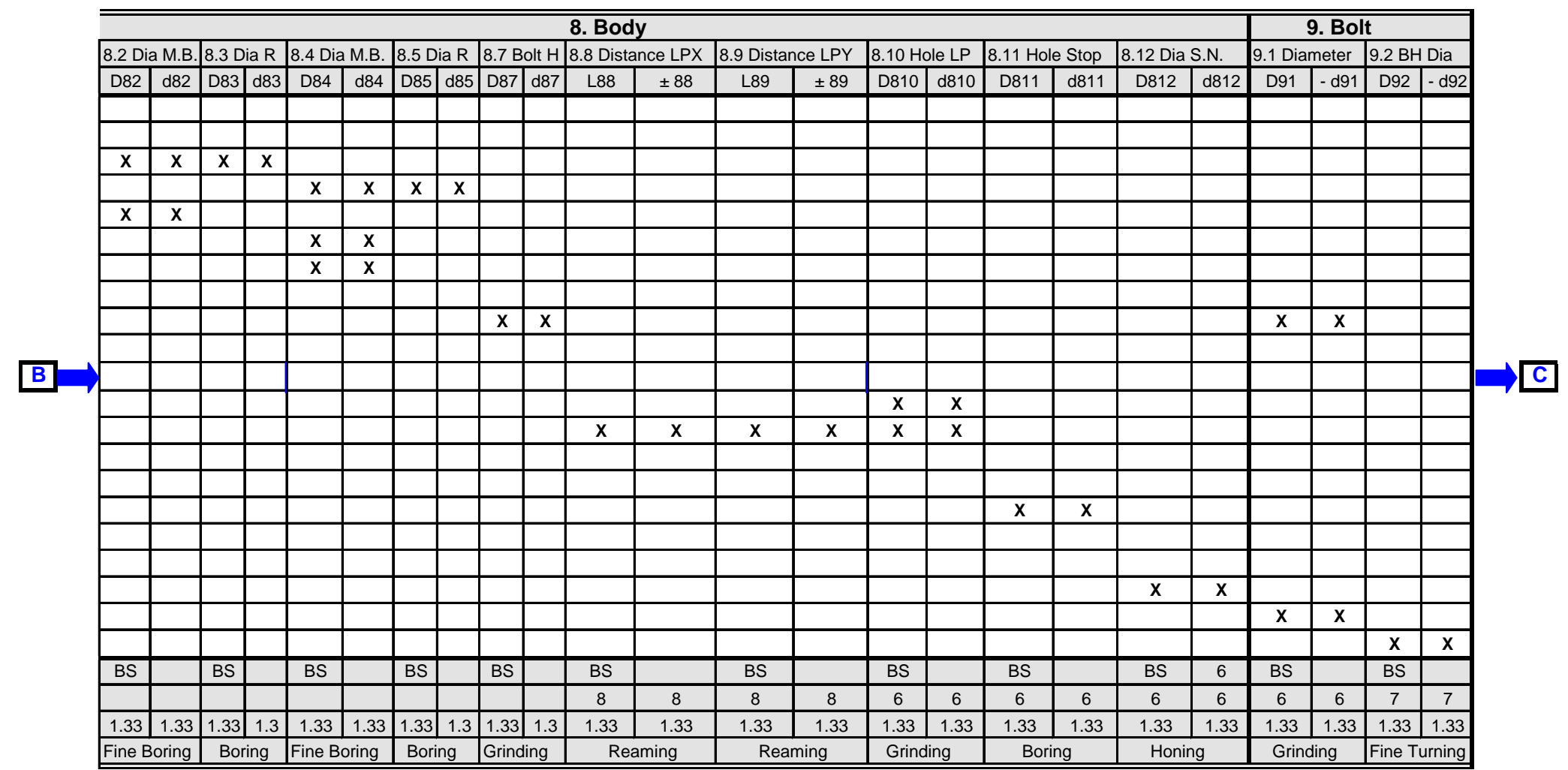

Figure 7 (Cotd). Complete DR/D Matrix 


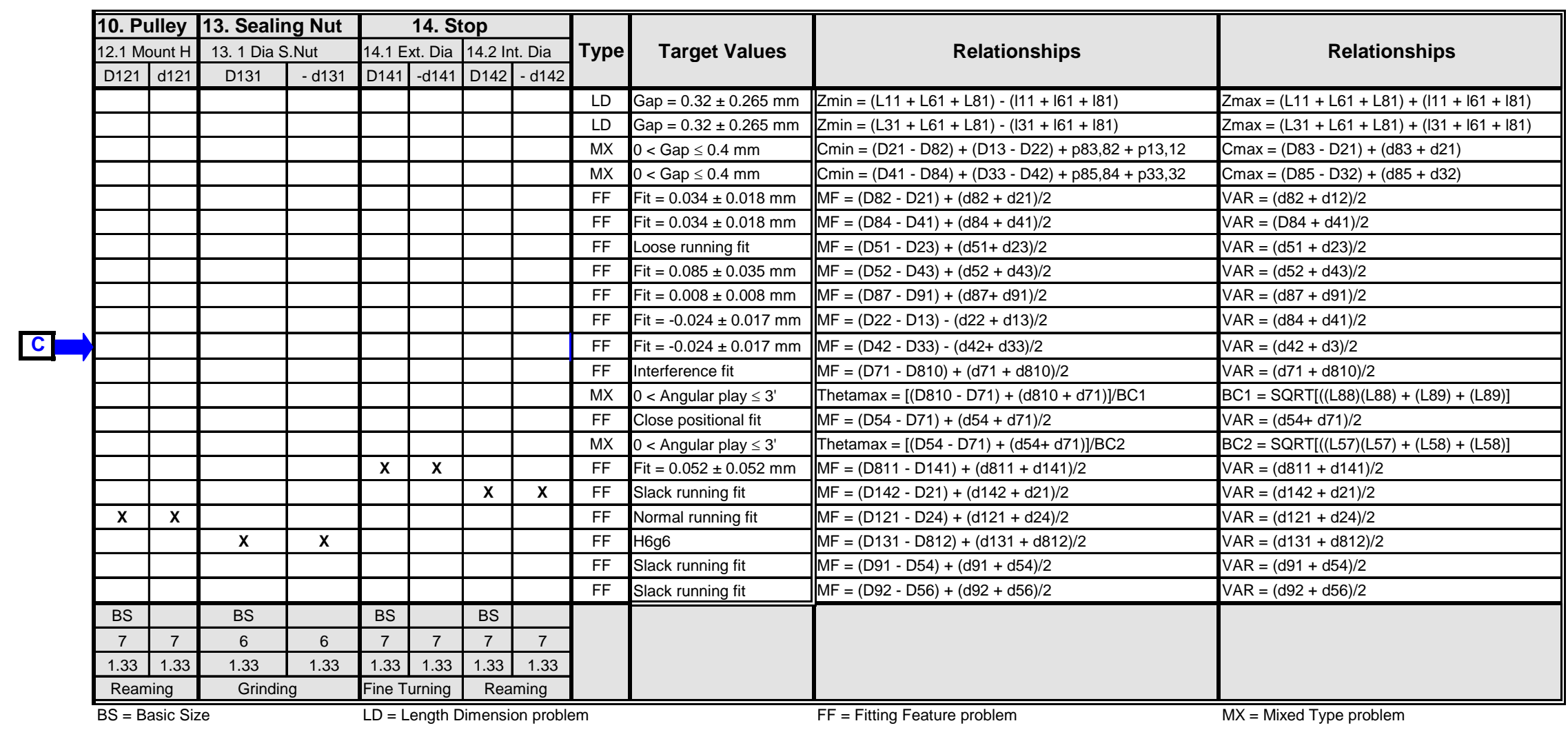

Figure 7 (Cotd). Complete DR/D Matrix 


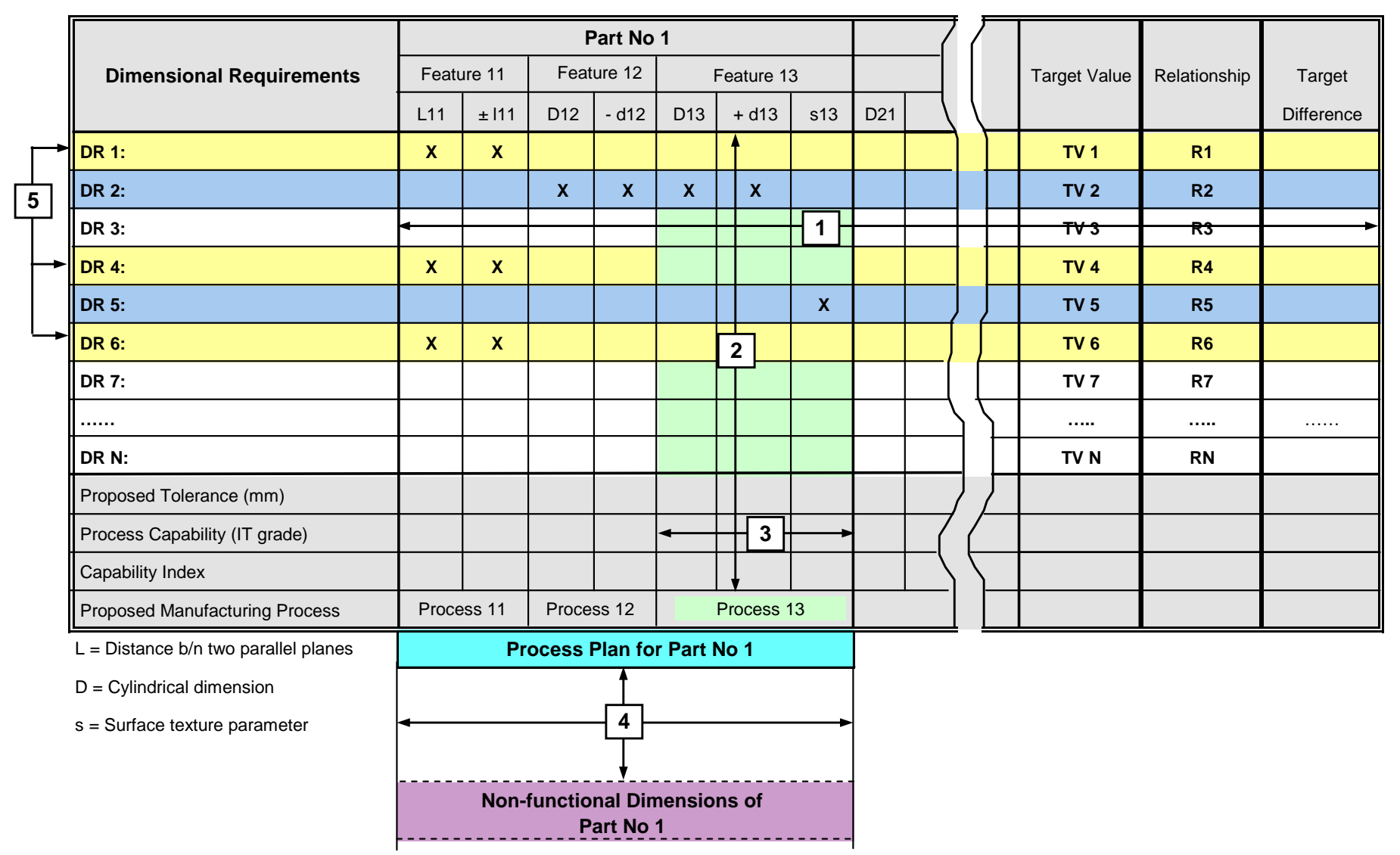

Figure 8. Types of Relationships Illustrated in DR/D Matrix 